\title{
Rate of Hanger Reflex Occurrence: Unexpected Head Rotation on Fronto-temporal Head Compression
}

\author{
Takashi AsAHI, ${ }^{1}$ Michi SATO, ${ }^{2}$ Hiroyuki KaJIMOTO, ${ }^{2}$ Masaki KoH, ${ }^{1}$ \\ Daina KASHIWAZAKI, ${ }^{1}$ and Satoshi KURODA ${ }^{1}$
}

\author{
${ }^{1}$ Department of Neurosurgery, Graduate School of Medicine and Pharmaceutical Science, \\ University of Toyama, Sugitani, Toyama; \\ ${ }^{2}$ Department of Informatics, The University of Electro-Communications, Chofu, Tokyo
}

\begin{abstract}
When the head is encircled with a wire clothes hanger and the unilateral fronto-temporal region is compressed, the head rotates unexpectedly. As the mechanism is unclear, however, we have temporarily named this phenomenon as the "hanger reflex." We previously reported a case wherein this phenomenon was applied to treat cervical dystonia. Because little is known about this phenomenon, we determined how often this phenomenon is observed in healthy subjects. Study participants were 120 healthy Japanese adults (60 men and 60 women) aged 19-65 years. A wire clothes hanger was applied to each subject's head. The longer side of the hanger was attached over the volunteer's fronto-temporal regions on both sides of the head in succession (i.e., two applications per volunteer). We evaluated whether the subjects felt the sensation of head rotation by using a questionnaire. The sensation of head rotation was observed in $95.8 \%$ of subjects. There were five non-responders $(4.2 \%)$. In $85.4 \%$ of the trials, head rotation was observed in the direction that coincided with the side compressed by the hanger. There were no differences in responses between genders. The incident rate of the hanger reflex was remarkably high and most likely represents a prevalent phenomenon in humans. The mechanism underlying the reflex remains unknown. Further research should be performed to elucidate the underlying causes of the hanger reflex, which represents a potential treatment for cervical dystonia.
\end{abstract}

Key words: hanger reflex, cervical dystonia, neuromodulation

\section{Introduction}

When the head is encircled by an ordinary wire clothes hanger (Fig. 1A) and the fronto-temporal region is compressed by the longer side of the hanger, head rotation towards the compressed side occurs unexpectedly (Fig. 1B). Although we have investigated this phenomenon, the underlying mechanism is not known. ${ }^{1-3)}$ We have tentatively named this phenomenon the "hanger reflex."1-3) A similar phenomenon was reported during the treatment of cervical dystonia using a cardboard box, ${ }^{4)}$ but this study was largely unnoticed. We stimulated the hanger reflex in a cervical dystonia patient and found that wearing a hanger restricted head rotation. We invented a portable device to induce the hanger reflex and applied it to a patient with cervical dystonia (Fig. 2A, B). ${ }^{3}$ The possibility of a novel treatment for dystonia using this

Received September 20, 2014; Accepted November 14, 2014 phenomenon was thus re-realized. Presently, the etiology and epidemiology of the hanger reflex are not well known. We investigated the occurrence rate of the hanger reflex to determine how often it is observed in healthy subjects.

\section{Materials and Methods}

Subjects: One hundred and twenty healthy Japanese adults (60 men and 60 women) aged 19-65 years participated in this study. These subjects were recruited from staff and students at the University of Toyama and the University of ElectroCommunications. Informed consent was obtained from all the subjects.

Methods: A hanger was applied to each subject's head. This steel wire hanger is commonly used in Japan, and has a width of $30.5 \mathrm{~cm}$ and a height of $11 \mathrm{~cm}$ (Fig. 1A). The wire was flexible and could be applied to the head. Instructions on how to apply the hanger to the head were distributed to 

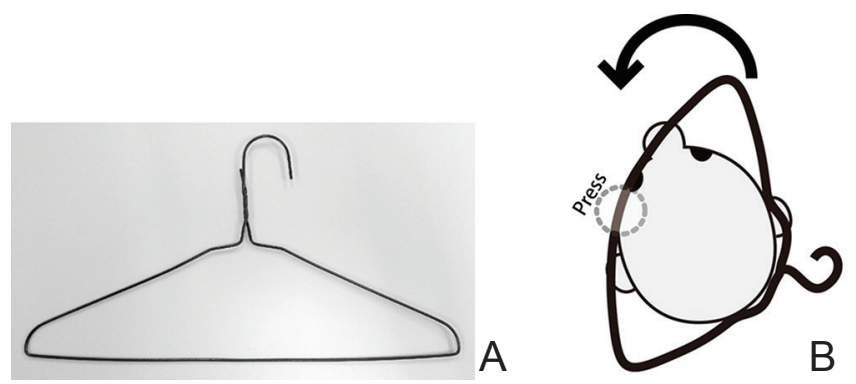

Fig. 1 Diagram of the hanger reflex. A: Hanger used for this study. This is an ordinary wire clothes hanger commonly available in Japan. It is flexible and could surround the head. B: When the head is encircled within an ordinary wire clothes hanger, and its fronto-temporal region is compressed, head rotation to the compressed side unexpectedly occurs.

the subjects before the trials. These instructions included pictures and indicated the key point to be compressed. Two experimental sequences, either left first or right first compression, were used. The order was assigned randomly to the subjects by a double-blinded investigator. In order to prevent a bias of the responses to either the first or the second trial, half of both male and female subjects were assigned to the left first trial. The other half started with the right first trial. The longer side of the hanger was attached over the fronto-temporal region on either side of each subject's head in succession (i.e., two applications per volunteer). The subjects attached the hanger themselves according to the provided instructions. While wearing the hanger, they slowly rotated their head bilaterally. The subjects then answered questionnaires, which included queries on age, gender, and whether they felt the head rotation sensation in each trial. If they felt the sensation, they specified in which direction it occurred. They repeated the procedure on the opposite side according to the same instructions. The subjects were not instructed as to which direction the head was predicted to rotate. This study was reviewed and then approved by our institutional ethics committee.

Statistical analysis: Pearson's chi-square tests were applied to evaluate the differences in responses between both the first and the second trials and also between genders.

\section{Results}

Table 1 shows the response patterns for each subject. They were divided into six patterns. Seventy-nine point two percent of the subjects experienced bilateral head rotation toward the compressed side (A).
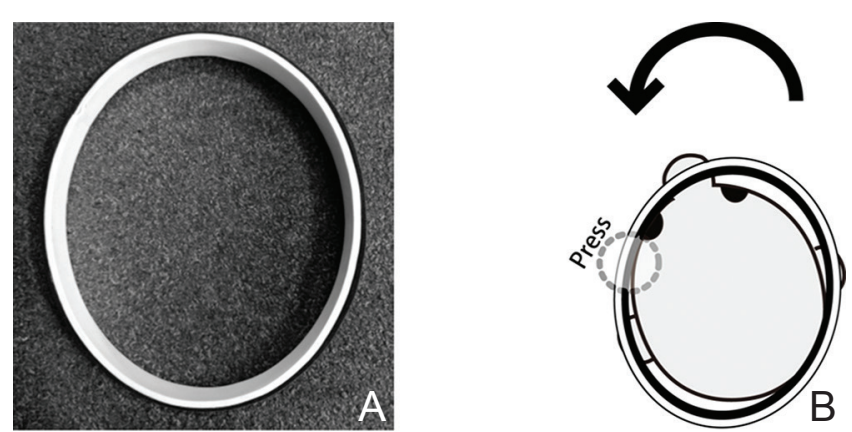

Fig. 2 A portable device inducing the hanger reflex. A: This elliptical-shaped device is very light and coated with urethane to prevent local pain. $B$ : If the device is rotated, the fronto-temporal head region is compressed and the hanger reflex is induced. If the patient wears a cap, it is invisible and cosmetically acceptable.

Table 1 Response patterns for each subject. Six response patterns were observed

\begin{tabular}{lrr}
\hline & \multicolumn{1}{c}{$\mathrm{n}$} & \multicolumn{1}{c}{$\%$} \\
\hline (A) Bilaterally compressed side & 95 & 79.2 \\
(B) Unilaterally compressed side & 9 & 7.5 \\
(C) Unilaterally uncompressed side & 1 & 0.8 \\
(D) Bilaterally same side & 6 & 5.0 \\
(E) Bilaterally uncompressed side & 4 & 3.3 \\
(F) No response & 5 & 4.2 \\
\hline Total & 120 & 100 \\
\hline
\end{tabular}

Table 2 Response patterns of each trial. The responses from 240 trials were classified into three patterns

\begin{tabular}{lrr}
\hline & $\mathrm{n}$ & $\%$ \\
\hline Compressed side & 205 & 85.4 \\
Uncompressed side & 16 & 6.7 \\
No rotation & 19 & 7.9 \\
\hline Total & 240 & 100 \\
\hline
\end{tabular}

Seven point five percent experienced rotation toward the compressed side, but on only one side of the head (B). One case $(0.8 \%)$ reported rotation opposite to the compressed side on only one side of the head (C), $5.0 \%$ experienced rotation in the same direction when the head was compressed on either side (D), and $3.3 \%$ exhibited rotation opposite to the compressed side, bilaterally (E). Five cases (4.2\%) experienced no rotation sensations at all (F). Tables 2-4 summarize the rotation patterns, defined as follows: (1) head rotation toward the compressed side, (2) head rotation toward the uncompressed side, and (3) no head rotation. Table 2 displays a summary of the 
Table 3 Responses divided into trial orders. There was no significant differences among them $(p=0.441)$

\begin{tabular}{lccc}
\hline & First trial & Second trial & Total \\
\hline Compressed side & 99 & 106 & 205 \\
Uncompressed side & 9 & 6 & 15 \\
No rotation & 12 & 8 & 20 \\
\hline Total & 120 & 120 & 240 \\
\hline
\end{tabular}

Table 4 Responses divided into genders. There was no statistical differences $(p=0.247)$

\begin{tabular}{lrrc}
\hline & Male & Female & Total \\
\hline Compressed side & 107 & 98 & 205 \\
Uncompressed side & 6 & 9 & 15 \\
No rotation & 7 & 13 & 20 \\
\hline Total & 120 & 120 & 240 \\
\hline
\end{tabular}

responses for each trial. Each subject underwent two trials. Therefore, 240 trials were conducted in total. Rotation sensations occurred in 92.1\% (compressed side: $85.4 \%$, uncompressed side: $6.7 \%$ ). No rotation occurred only in $7.9 \%$ of subjects.

Table 3 shows differences between the first and second trial. The first trial means the first one of two trials assigned to either right or left compression. There was no significant difference related to the order of right versus left trials $(p=0.441)$. Table 4 shows a summary of responses divided according to gender. There was no statistical difference in responses between them $(p=0.247)$.

\section{Discussion}

The sensation of head movement when the frontotemporal region was compressed occurred frequently in healthy subjects (Table 1A-E; 110/120, 95.8\%). The head of the majority of subjects $(79.2 \%)$ rotated bilaterally to the compressed side (Table 1A) and there were few non-responders $(4.2 \%)$ (Table $1 \mathrm{~F}$ ). There were clear response patterns. However, we could not elucidate any differences in these response patterns that might depend on the asymmetry of individual head shape and/or head size. As for the analysis focused on the each individual trial, more than $90 \%$ of the subjects felt a rotating sensation of the head (Table 2). The majority of them experienced rotation to the compressed side (85.4\%). In this study, we did not instruct the subjects as to which direction the head was supposed to be rotated. This rate of occurrence is quite high, which means head rotation to the compressed side cannot be explained by a placebo effect. Furthermore, there was no statistical difference in responses between left versus right trial order (Table 3). We could not find statistical differences in responses between genders (Table 4). Taken together, our results demonstrate that the hanger reflex frequently occurs in healthy male and female subjects and we verified that this is not a placebo effect. In this study, we did not quantify the angle of head rotation, because no previous studies described it. However, the angle of head rotation should be quantified to clarify the mechanism through which the hanger reflex induces head rotation, in future studies.

Christensen applied a square cardboard box fitted to the heads of the two spasmodic torticollis patients at approximately $45^{\circ}$ and the heads were rotated to push on the fronto-temporal forehead; abnormal head rotation was restricted.4) Additionally, 9 out of 10 healthy volunteers experienced head rotation in response to pressure from the cardboard box. In 1995, a young man found that when he wore a wire clothes hanger on his head, his head rotated involuntarily. This fact was broadcasted on a TV program in Japan. After a decade, Kajimoto and a co-researcher studied this phenomenon scientifically. ${ }^{1,2)}$ Matsue et al. demonstrated that the reflex was induced by compression on the fronto-temporal region without pain sensation. ${ }^{1)}$ They developed a special machine that involuntarily rotates the head by just pushing on the fronto-temporal region. ${ }^{2}{ }^{2}$ They found that compression without pain sensation is necessary for generating the reflex.

Recently, we monitored an electromyogram (EMG) activity of the sternocleidomastoid muscle (SCM) during hanger reflex in normal subjects. As the results, the application of a hanger onto the frontotemporal region suppressed the EMG activity of the ipsilateral SCM and simultaneously increased that of the contralateral SCM with their head rotation. In a patient with cervical dystonia, the hanger reflex suppressed the abnormal EMG activities of the inferior obliquus capitis muscle and improved the patient's symptoms. ${ }^{5}$ We do not think that the hanger reflex is a sensory trick which is well known as a phenomenon that temporarily relieves the symptoms of dystonia, because hanger reflex, but not sensory trick, induce even normal subjects. Hanger reflex does not require visual information according to previous studies. ${ }^{1,2)}$ For now, we hypothesize that skin shearing force to the rotation side may cause the involuntary head rotation. We have found a similar phenomena in the other regions of the body, including the wrist and waist. ${ }^{6}{ }^{6}$ Therefore, hanger reflex is considered as one of the universal phenomena in the body. 
In the ongoing clinical trial, the hanger reflex can be observed in most of the patients with cervical dystonia as well as in normal subjects (unpublished data). ${ }^{7)}$ Most importantly, the hanger reflex can easily be induced when the ideal angles of the hanger triangle appropriately compresses the temporal line of the skull, which can maximally provoke skin shearing force to the compressed side.

As mentioned above, we found that the abnormal head rotation in cervical dystonia patients was restricted by the hanger reflex. However, wearing a typical clothes hanger while walking is unrealistic. Therefore, we invented a small portable device made with gypsum that is designed to generate sufficient pressure to the fronto-temporal region by rotating it (Fig. 2A, B). ${ }^{8)}$ The device is elliptical and the inside of the device is coated with urethane to prevent local pain. It is very light and invisible if the patient wears a cap. We applied it to a patient with cervical dystonia, and his head rotation was then restricted while wearing the device. ${ }^{3)}$ After applying the device for 1 month, the degree of head rotation was restricted even without wearing the device. This finding seems to be similar to that reported in a previous article. ${ }^{4)}$

A variety of treatments are performed for cervical dystonia, including electrical stimulation, ${ }^{9)}$ bio feedback, ${ }^{10)}$ physical therapy, ${ }^{11,12)}$ botulin injection, ${ }^{13)}$ spinal cord stimulation, ${ }^{14)}$ deep brain stimulation (DBS), ${ }^{15-17)}$ and selective nerve denervation. ${ }^{18)}$ According to the guidelines of the European Federation of Neurological Societies, intramuscular Botulinum toxin (BTX) injection can be regarded as a first-line treatment for cervical dystonia. ${ }^{19)}$ BTX treatment was beneficial in $43-68 \%$ of subjects compared to $10-22 \%$ of the placebo group. ${ }^{20)}$ However, the treatment requires repeated injections every 3 months and it was not available to some patients, because of its cost. DBS is recommended for patients who do not respond to BTX. ${ }^{15-17)}$ The targets are the globus pallidus (GPi) or subthalamic nucleus (STN). In the GPi DBS cases, a long-term (mean $=31.9$ months, range $=12-67$ months) follow-up study showed severity and disability subscores of the Toronto Western Spasmodic Torticollis Rating Scale (TWSTRS) improved by $54.8 \%$ and $59.1 \%$, respectively. ${ }^{16)}$ In a study of STN-DBS, it was found that the severity scale score of TWSTRS improved by $61 \% .{ }^{17)}$ Thus, treatment options for dystonia are invasive and some patients do not respond to these treatments. In light of our findings, a multicenter clinical trial utilizing the hanger reflex is ongoing in Japan to evaluate its efficacy in treating cervical dystonia. ${ }^{7)}$ The hanger reflex has potential to be a novel, less invasive, and inexpensive treatment for cervical dystonia.

\section{Conclusion}

We found the hanger reflex is highly and equally prevalent in healthy subjects of both sexes. This phenomenon may be useful in treating cervical dystonia patients. However, there is little evidence of what factors are needed to induce the hanger reflex, and the etiology of the phenomenon is still unknown. Further research is required to estimate the therapeutic potential of the hanger reflex.

\section{Conflicts of Interest Disclosure}

This study was supported by JSPS KAKENHI (23791587) and a Hokugin grant for young scientists. The JSPS KAKENHI and Hokugin grant for young scientists provided unrestricted support and had no role in the oversight or review of the research data or reporting. The authors have no conflicts of interest to declare.

\section{References}

1) Matsue R, Sato M, Hashimoto Y, Kajimoto H: "Hanger reflex": A reflex motion of a head by temporal pressure for wearable interface. SICE Annual Conference 2008 1463-1467, 2008

2) Sato M, Matue R, Hashimoto Y, Kajimoto H: Development of a head rotation interface by using hanger reflex. 18th IEEE International Symposium on Robot and Human Interactive Communication 534-538, 2009

3) Asahi T, Hayashi N, Hamada H, Sato M, Kajimoto H, Takashima S, Endo S: Application of the Hanger Reflex to the treatment of cervical dystonia. Funct Neurosurg 49: 173-176, 2010 (Japanese)

4) Christensen JE: New treatment of spasmodic torticollis? Lancet 338: 573, 1991

5) Aiba A, Asahi T, Kajimoto H, Sato M, Oyama G, Taira T, Hayashi A: Botulinum toxin treatment of cervical dystonia with portable needle electromyography and a clinical trial of the hanger reflex. J Mov Disord Disabil 24: 13-18, 2014 (Japanese)

6) Nakamura $T$, Nishimura $N$, Sato $M$, Kajimoto $H$ : Application of Hanger Reflex to wrist and waist. Virtual Reality (VR), 2014 iEEE 181-182, 2014

7) UMIN Clinical Trials Registry. 2012. (Accessed at https://upload.umin.ac.jp/cgi-open-bin/ctr/ctr.cgi? function=brows\&action=brows\&type=summary\&rec ptno=R000009153\&language $=$ J.)

8) Asahi T, Sato M, Kajimoto H; inventors: A device for the treatment of cervical dystonia. Japanese patent No. 5552844, 2014

9) Leis AA, Dimitrijevic MR, Delapasse JS, Sharkey PC: Modification of cervical dystonia by selective 
sensory stimulation. J Neurol Sci 110: 79-89, 1992

10) Jahanshahi M, Sartory G, Marsden CD: EMG biofeedback treatment of torticollis: a controlled outcome study. Biofeedback Self Regul 16: 413-448, 1991

11) Zetterberg L, Halvorsen K, Färnstrand C, Aquilonius SM, Lindmark B: Physiotherapy in cervical dystonia: six experimental single-case studies. Physiother Theory Pract 24: 275-290, 2008

12) Smania N, Corato E, Tinazzi M, Montagnana B, Fiaschi A, Aglioti SM: The effect of two different rehabilitation treatments in cervical dystonia: preliminary results in four patients. Funct Neurol 18: 219-225, 2003

13) Poewe W, Deuschl G, Nebe A, Feifel E, Wissel J, Benecke R, Kessler KR, Ceballos-Baumann AO, Ohly A, Oertel W, Künig G: What is the optimal dose of botulinum toxin $\mathrm{A}$ in the treatment of cervical dystonia? Results of a double blind, placebo controlled, dose ranging study using Dysport. German Dystonia Study Group. J Neurol Neurosurg Psychiatry 64: 13-17, 1998

14) Waltz JM, Scozzari CA, Hunt DP: Spinal cord stimulation in the treatment of spasmodic torticollis. Appl Neurophysiol 48: 324-338, 1985

15) Kiss ZH, Doig-Beyaert K, Eliasziw M, Tsui J, Haffenden A, Suchowersky O; Functional and Stereotactic Section of the Canadian Neurosurgical Society; Canadian Movement Disorders Group: The Canadian multicentre study of deep brain stimulation for cervical dystonia. Brain 130 : 2879-2886, 2007

16) Hung SW, Hamani C, Lozano AM, Poon YY, Piboolnurak P, Miyasaki JM, Lang AE, Dostrovsky JO, Hutchison WD, Moro E: Long-term outcome of bilateral pallidal deep brain stimulation for primary cervical dystonia. Neurology 68: 457-459, 2007

17) Ostrem JL, Racine CA, Glass GA, Grace JK, Volz MM, Heath SL, Starr PA: Subthalamic nucleus deep brain stimulation in primary cervical dystonia. Neurology 76: 870-878, 2011

18) Taira T, Hori T: A novel denervation procedure for idiopathic cervical dystonia. Stereotact Funct Neurosurg 80: 92-95, 2003

19) Albanese A, Asmus F, Bhatia KP, Elia AE, Elibol B, Filippini G, Gasser T, Krauss JK, Nardocci N, Newton A, Valls-Solé J: EFNS guidelines on diagnosis and treatment of primary dystonias. Eur J Neurol 18: 5-18, 2011

20) Snaith A, Wade D: Dystonia. BMJ Clin Evid 2011: pii 1211,2011

Address reprint requests to: Takashi Asahi, MD, PhD, Department of Neurosurgery, Graduate School of Medicine and Pharmaceutical Science, University of Toyama, Sugitani 2630, Toyama 930-0194, Japan. e-mail: takashi-tym@umin.ac.jp 